\title{
Ebola Virus L Polymerase RdRp Sequence and Phylogenetic Analysis
}

\author{
M.I. El Gohary*, Abdo A. Elfiky**, A. Eissa, and Amr.A. Desouky \\ Physics Department, Faculty of Science (Boys), Al-Azhar University, Nasr City, Cairo, \\ **Biophysics Department, Faculty of Science, Cairo University, Giza, Egypt
}

\begin{abstract}
$\mathbf{E}$ BOLA Virus (EBOV) infection affects humans beings in the last four decades with the deadliest outbreak at 2015 in West Africa, leaving more than 10,000 deaths. The virus harms the liver of the patient when direct contact with contaminated body fluids or blood is occurred. $\mathrm{L}$ polymerase is one of the viral proteins responsible for the viral RNA replication. Inhibition of the viral polymerase succeeded is in stopping the infection of other viruses such as Hepatitis C Virus (HCV). National Center for Biotechnology Information (NCBI) protein database has 2123 sequences for L polymerase. In present research, the sequence and phylogenetic analysis are utilized to understand the non-redundant sequence coming from different countries. Based on the sequence similarity, the solved structure of vesicular stomatitis virus (PDB ID: 5A22) is used in this work to suggest the active site of the EBOV RdRp domain. Two newly released sequences (APT69557.1 and ALX33626.1 for the Sudan and Zaire, respectively) are based on the phylogenetic analysis, show interestingly a divert, mutation and distance from its subgroups suggesting a new emerged isoform of EBOV RdRp. The active site motif, GDN, would be targeted by polymerase inhibitors succeeded in other viruses to get stop the infection.
\end{abstract}

Keywords: L polymerase, EBOV, GDN motif, phylogenetic analysis, sequence alignment.

\section{Introduction}

Ebola virus is stand out amongst the most harmful pathogens known to contaminate people. ${ }^{1}$ The first recognized Ebola outbreak occurred at 1976, near Ebola River in Zaire (now Democratic Republic of Congo, DRC). In recent years, more than 20 flare-ups have happened in Africa, with the vast majority of the known episodes happenned in the previous 20 years. $^{2}$

The main routes of Ebola virus transmission are direct contact with asymptomatic Ebola patient's blood and body fluids (including but not restricted to urine, feces, vomitus, saliva, and sweat) through breaks in the skin or inoculation into the mouth, nose or eyes. Human contamination can likewise happen through contact with wild animals, such as by hunting, butchering or preparing meat from infected animals ${ }^{3}$. Ebolavirus (EBOV) causes an exceptionally infectious zoonotic disease, affects humans and other primates. Although the natural outbreak of the EBOV is yet restricted to Africa, fast methods for individual's correspondence, high viral transmissibility, and high mortality rate have made the EBOV a serious global health threat. Currently, there is no effective direct acting
anti-EBOV drug. EBOV patients receive only palliative therapy. ${ }^{4}$

Currently, there are no licensed vaccines or treatments available to combat EBOV disease and as such, research aimed at identifying targets for therapeutic intervention is of high priority. However, the classification of EBOV as a biosafety level 4 (BSL-4) pathogen greatly limits studies using a live virus. ${ }^{5}$ Nearly every Ebola virus protein has been characterized for therapeutic targeting potential. ${ }^{6}$

The EBOV genome is a negative-sense singlestranded RNA and contains a viral envelope, matrix, and nucleocapsid components. It encodes seven structural proteins: nucleoprotein (NP), polymerase cofactor (VP35, VP40, GP), transcription activator (VP30, VP24), and RNAdependent RNA polymerase (L)., ${ }^{7,8}$ Viral RNAdependent RNA polymerases (RdRp) are essential for replication of RNA viruses and represent important drug targets. ${ }^{9}$

CLUSTAL $\Omega \mathbf{1 0}^{\mathbf{1 0}}$ is web -based software service for performing fast and accurate multiple sequence alignments (MSAs) of potentially large 
numbers of protein or DNA/RNA sequences. It is the latest version of the famous and widely used CLUSTAL MSAs . ${ }^{11,12}$.

The aim of present work to determine the active site of RdRp of EBOV to use in docking method with anti-viral drugs. Study the relationship of EBOV L polymerase sequence by pairwise distance and phylogenetic tree.

\section{Materials and Methods}

\section{Multiple sequence alignment}

Multiple sequence alignment was performed using the web server CLUSTAL $\Omega{ }^{10}$. The alignment was ordered and represented using Easy Sequencing in PostScript (ESPript 3.0) web server ${ }^{13,14}$. ESPript 3.0 generates figures of aligned sequences with secondary structure information. It can serve as a tool for structure/ function analyses. ESPript reads text outputs from multiple sequence alignment programs such as CLUSTAL $\Omega$ and MULTALIN, as well as from programs able to identify secondary structure elements from structure files such as $\operatorname{DSSP}^{15}$ and STRIDE $^{16}$.

\section{EBOV polymerase Sequence analysis}

2123 of EBOV $\mathrm{L}$ polymerase protein representing all recorded EBOV outbreaks as retrieved from the National Center for Biotechnology Information (NCBI) http:// www.ncbi.nlm.nih.gov/, ${ }^{17}$. EBOV L polymerase Sequence were downloaded from various countries (Sudan, Reston, Bombali, Zaire, Tai Forest and Bundibugyo) and synthetic construct (that Artificial viruses to understand and prevent viral disease).

\section{EBOV polymerase Sequence selected}

21 unique sequences for EBOV L polymerase protein were selsected. Eight from Sudan, three from Reston (United States), two from Bombali (Sierra Leone), three from Zaire, two from Tai Forest (Côte d'Ivoire), two from Bundibugyo (Uganda) and one synthetic construct.

Pairwise distance method and (MEGA) software

Pairwise distance method is used to test the distances between the aligned sequences using Molecular Evolutional Genetics Analysis (MEGA) software ${ }^{18}$ in table 1.

\section{Phylogenetic tree}

Phylogenetic tree of the aligned sequences is also calculated using MEGA software and represented by the Cladogram.

\section{$\underline{\text { Results and Discussion }}$}

Sequence alignment

To obtain the correct alignment of a large set of sequences, with some of them being only distantly related, it is essential to elaborate an optimal scheme for hierarchical alignment and to delimit the portions of the sequences which are optimal for revealing non-random similarity. Sequences of various sets of negative-strand viral RNA of Ebola polymerases have been downloaded from NCBI http://www.ncbi.nlm.nih.gov/, ${ }^{17}$., The authors used CLUSTAL $\Omega$ website ${ }^{10}$ to perform multiple sequence alignment of the 21 sequences of RdRp of EBOV L polymerase. The total number of downloaded sequences are 2123 according to various countries (Sudan, Reston, Bombali, Zaire, Tai Forest, Bundibugyo) and synthetic construct.

After reducing the sequences for the $\mathrm{RdRp}$ domain, the number of non-redundant sequences becomes 21. The alignment is represented by ESPript 3.0 as shown in Fig.1. The alignment consists of 1082 amino acids and includes distinct blocks of amino acid residues which could be considered conserved motifs. Overall the sequence similarity is high (highlighted in red in Fig.1). The highest sequence identity is $99.91 \%$, and the lowest is $80.74 \%$. The secondary structure of the cryo-EM solved L protein of vesicular stomatitis virus (PDB ID: 5A22) is represented in the top of the alignment. This solved structure represents the best homolog for building the 3D model of EBOV RdRp (89 \% coverage and sequence Identity $17.4 \%$ ). The sequence of the solved structure (PDB ID: 5A22) is aligned against EBOV sequences. Based on the multiple sequence alignment, GDN motif (the reported active site of $\mathrm{L}$ protein of vesicular stomatitis virus) is conserved in EBOV with the two aspartate residues protruding from the beta-turn structure (between $\beta 13$ and $\beta 14$ ). This is suggested to be the active site for EBOV RdRp.

\section{Pairwise distance analysis}

The pairwise distance for the aligned sequences is represented in table 1. Pairwise distance, that is the minimum number of changes necessary to convert one sequence into another, for the sequences from the same country show shorter values, while the distances increase when aligning two sequences from different countries. Interestingly, Sudan EBOV shows the shortest distances compared to other EBOV sequence under the study. This implies the phylogenetic relevance as reported from the phylogenetic tree shown in Fig. 2. 

AGL50931.1:11-1090Sudan APT69557.1:11-1090Sudan AGL73449.1:11-1090Sudan AFP28229.1:11-1090Sudan AAA79970.1:11-1090Sudan AGB56682.1:11-1090Sudan ACR33194 . 1:11-1090Sudan ALL26379.1:11-1090Sudan AAV48581.1:10-1089Reston BAB69010.1:10-1089Reston AAN04454 . 1:10-1089Reston ASJ82200.1:10-1089Bombali ASJ82206.1:10-1089Bombali ALX33626.1:10-1089Zaire ARG43478.1:10-1089other AIR94011.1:10-1089Zaire ALX28268.1:10-1089Zaire ALT19766. 1:10-1089Tai ACI28636.1:10-1089Tai AGL73456.1:10-1089Bundibugyo ALT19775.1:10-1089Bundibugyo $\mathrm{pdb} / 5 \mathrm{~A} 22$ |A : 38-1070

$\mathrm{pdb} \mid 5 \mathrm{~A} 22$ |A : 38-1070

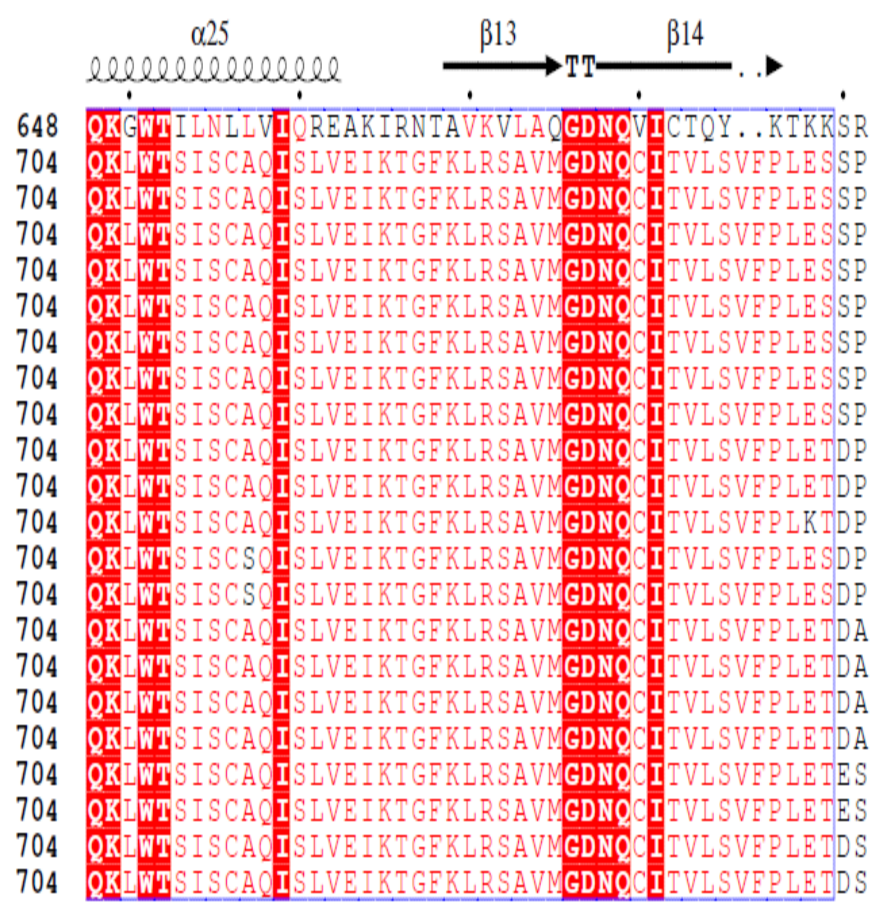

$\mathrm{pdb} / 5 \mathrm{~A} 22$ |A : 38-1070

pdb|5A22 |A : 38-1070 AGL50931.1:11-1090Sudan APT69557.1:11-1090Sudan AGL73449.1:11-1090Sudan AFP28229.1:11-1090Sudan AAA79970.1:11-1090Sudan AGB56682.1:11-1090Sudan ACR33194.1:11-1090Sudan ALL26379. 1:11-1090Sudan AAV48581.1:10-1089Reston BAB69010.1:10-1089Reston AAN04454.1:10-1089Reston ASJ82200.1:10-1089Bombali ASJ82206.1:10-1089Bombali ALX33626.1:10-1089Zaire ARG43478.1:10-1089other AIR94011.1:10-1089Zaire ALX28268.1:10-1089Zaire ALT19766. 1:10-1089Tai ACI28636.1:10-1089Tai AGL73456.1:10-1089Bundibugyo ALT19775. 1:10-1089Bundibugyo

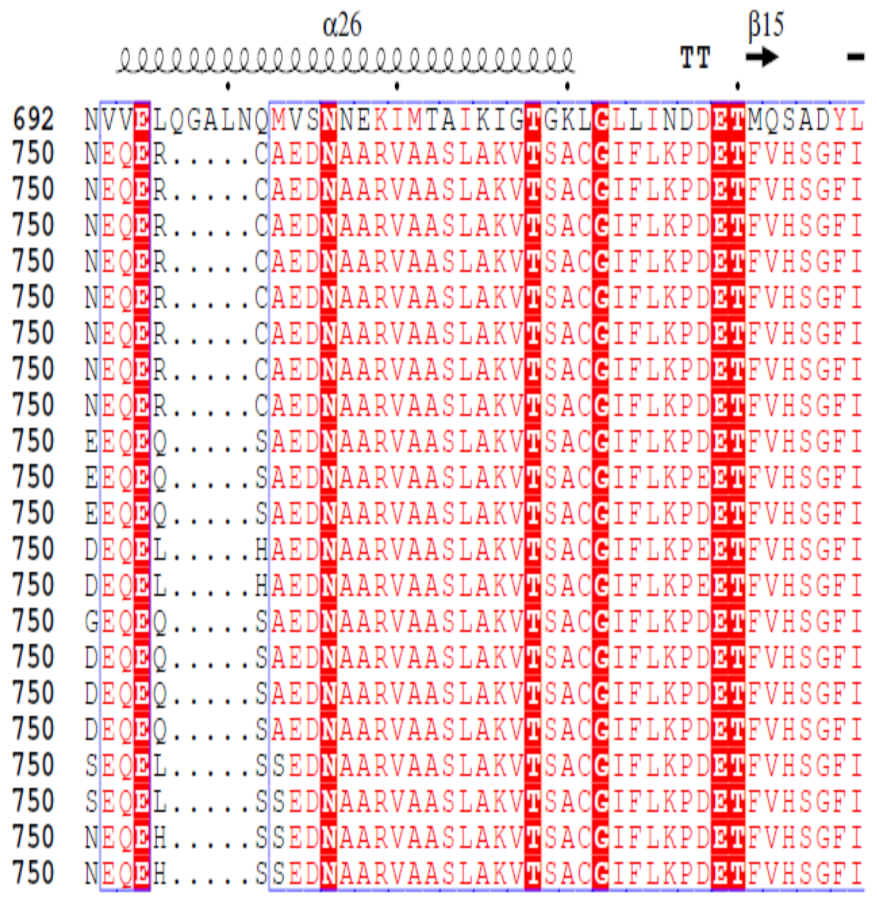

Egypt. J. Biophys. Biomed. Eng., Vol. 20 (2019) 
pdb / 5A22/A: 38-1070

pdb $\mid 5 A 22$ |A : 38-1070

AGL50931.1:11-1090Sudan

APT69557 . 1:11-1090Sudan

AGL73449.1:11-1090Sudan

AFP28229.1:11-1090Sudan

AAA79970.1:11-1090Sudan

AGB56682.1:11-1090Sudan

ACR33194 . 1:11-1090Sudan

ALL26379. 1:11-1090Sudan

AAV48581.1:10-1089Reston

BAB69010.1:10-1089Reston

AAN0 4454.1:10-1089Reston

ASJ82200 . 1:10-1089Bombali

ASJ82206. 1:10-1089Bombali

ALX33626.1:10-1089Zaire

ARG43478.1:10-1089other

AIR94011.1:10-1089Zaire

ALX28268. 1:10-1089Zaire

ALT19766.1:10-1089Tai

ACI28636. 1:10-1089Tai

AGL73456.1:10-1089Bundibugyo

ALT19775 . 1:10-1089Bundibugyo $\alpha 1$

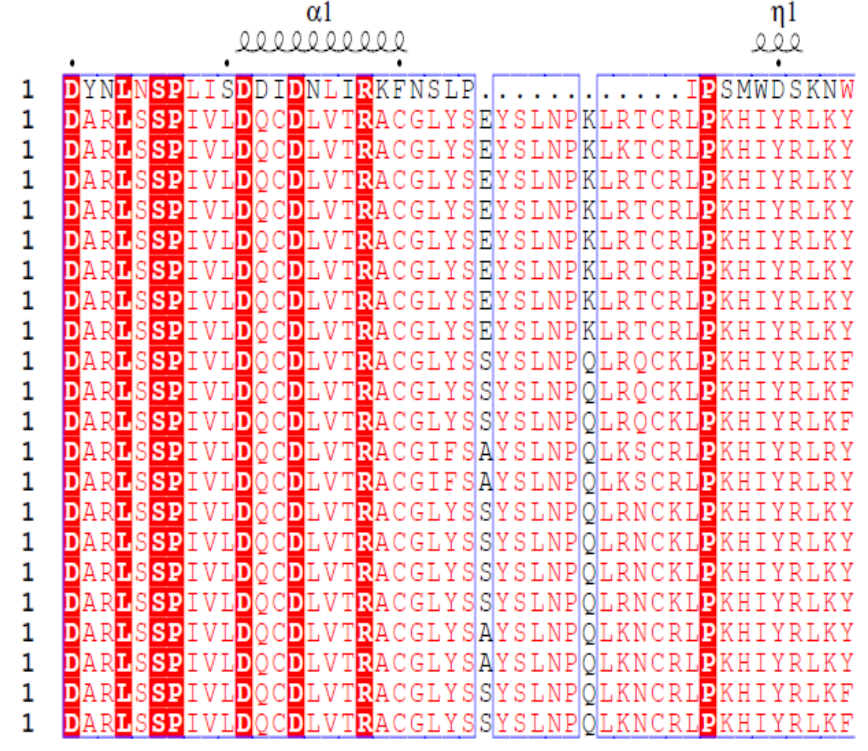

$\alpha 2$

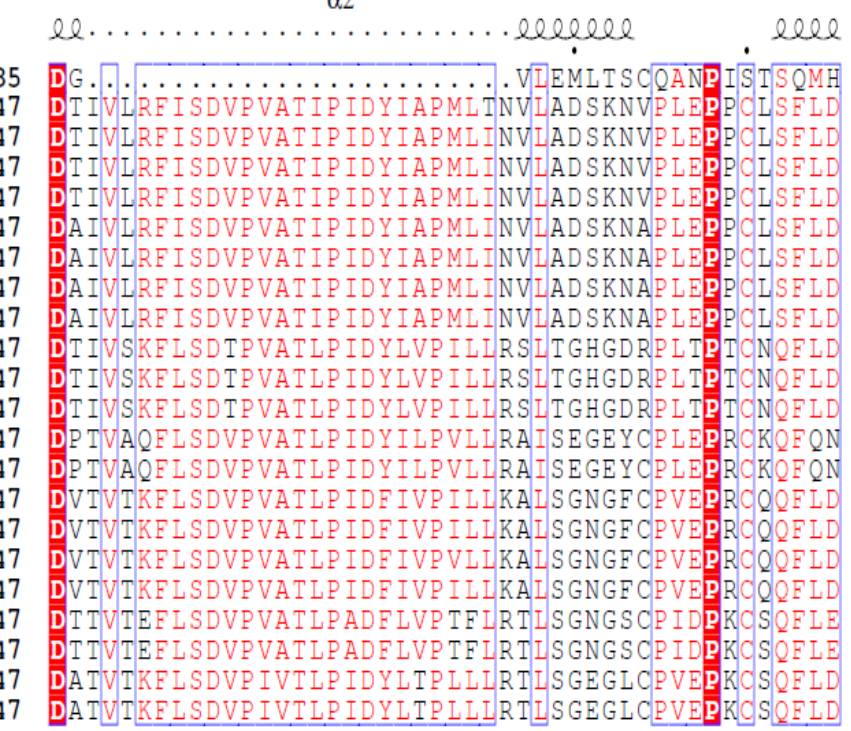

Fig.1. Sequence alignment for the 21 RdRp Ebola virus. The alignment is performed using CLUSTAL omega web server and visualized using ESPript software 3.0. The conserved amino acids are highlighted in red. The active site environment amino acids GDN are all conserved. 


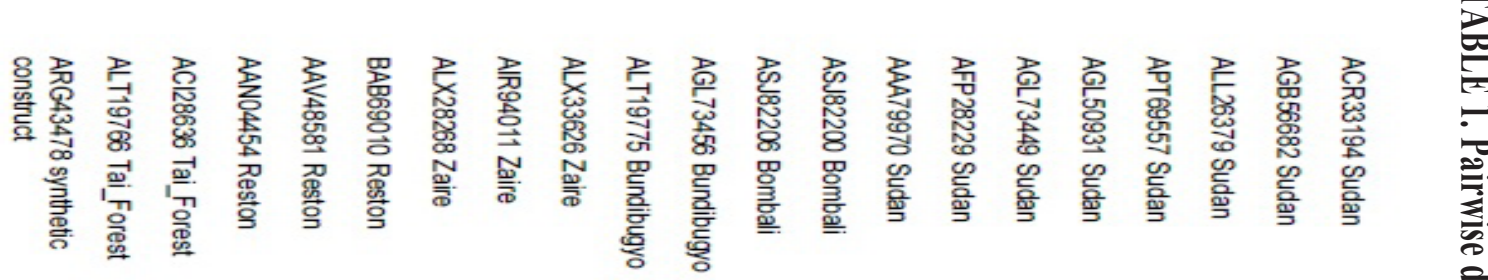

잉 공 芩

용용 兽

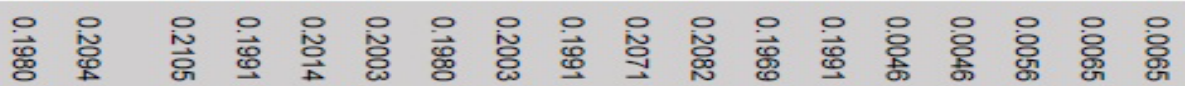

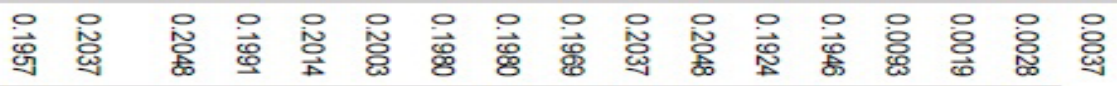

잉 兽 芯 芯 芯

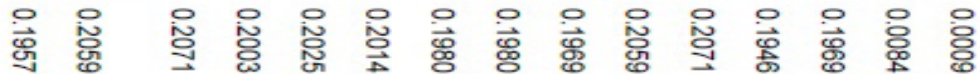

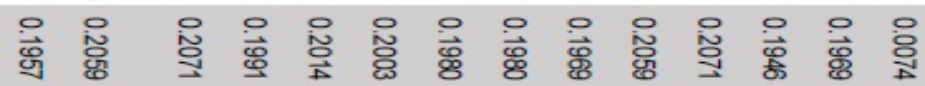

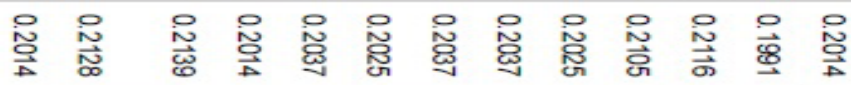

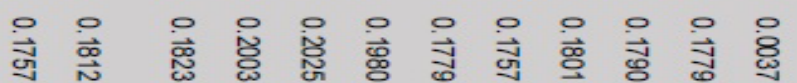

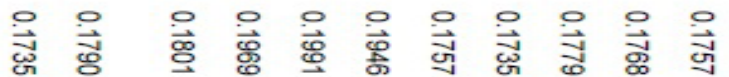

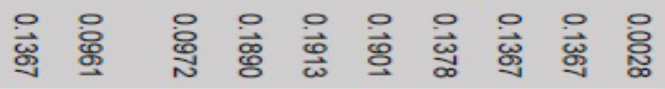

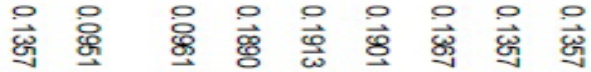

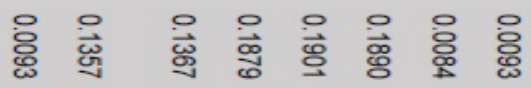

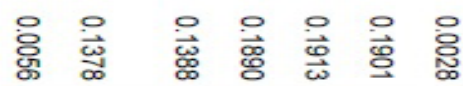

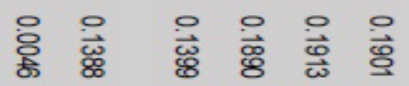

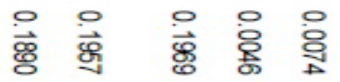

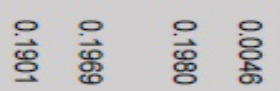

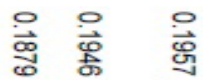

잉

$\stackrel{\circ}{\stackrel{\circ}{\leftrightarrows}}$ 


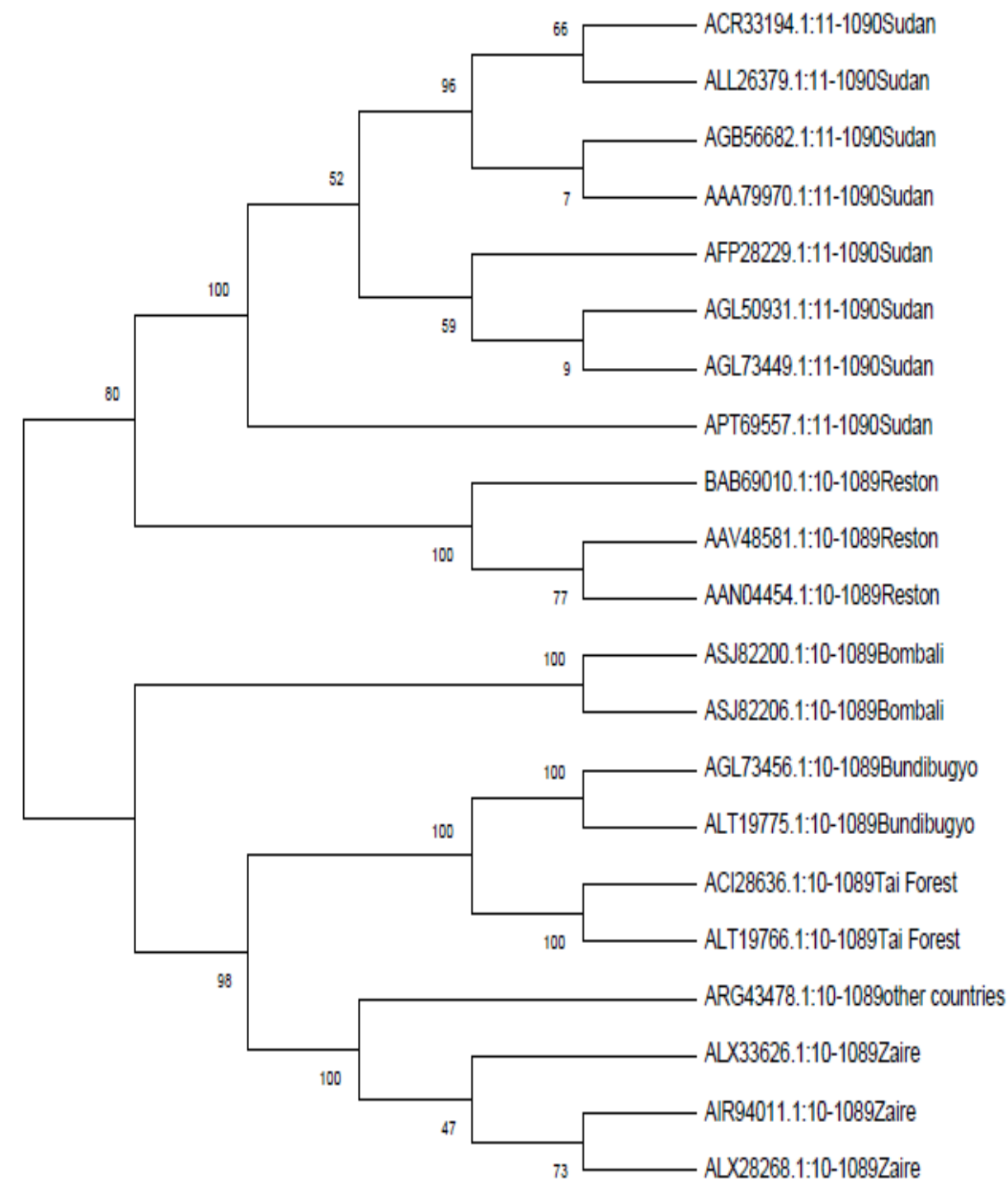

Fig. 2. A phylograph clarifying the evolutionary difference of RdRp Ebola virus. This phylogenetic tree is constructed using MEGA software. This graph is in the form of Cladograph.

\section{Phylogenetic tree}

Phylogenetic tree is inferred from the alignment as described in Materials and methods. A consensus tree inferred using the Maximum likelihood statistical method, bootstrap methods (test of phylogeny) with 100 replications, LG model and others with default parameters (Figure 2).

The aligned sequences of EBOV can be divided into two supergroup and branched into four lineages. The first lineage includes two subgroups; Sudan and Reston. The second group contains three subgroup; Bombali, Zaire and Tai Forest \& Bundibugyo. Synthetic construct lies in the same subgroup of Zaire which indicates that there are fewer mutations than the synthetic construct and Zaire sequences. Tai Forest and Interestingly, the Sudan APT69557.1 sequence that released in January 2017 showed that different lineage compared to other Sudan sequences published in the period (August 2002 to January 2016). The same result is also reported for Zaire Sequence ALX33626.1 which released in November 2018. This indicates a new emerged mutated isoform of the EBOV RdRp. 


\section{Conclusion}

EBOV RdRp active site is conserved among different sequence. The sequences analysis in the present study leads to RdRp inhibitors of other viruses such as $\mathrm{HCV}$ can be used to target EBOV L polymerase. Getting the 3D structure of EBOV L polymerase is the next step to test such polymerase inhibitors.

Among the observations of the phylogenetic analysis; sequences are occurred leading to distantly mutated isoforms and this should be as a target for future investigation.

\section{Reference}

1. Curtis N. Viral Haemorrhagic Fevers Caused by Lassa, Ebola and Marburg Viruses. 2006:35-44.

2. Kourtis AP, Appelgren K, Chevalier MS, McElroy A. Ebola Virus Disease. Pediatr Infect Dis J. 2015;34(8):893-897.

3. Falasca L, Agrati C, Petrosillo N, et al. Molecular mechanisms of Ebola virus pathogenesis: Focus on cell death. Cell Death Differ. 2015;22(8):1250-1259.

4. Teimoori S, Seesuay W, Jittavisutthikul S, et al. Human transbodies to VP40 inhibit cellular egress of Ebola virus-like particles. Biochem Biophys Res Commun. 2016;479(2):245-252.

5. Nelson E V., Pacheco JR, Hume AJ, et al. An RNA polymerase II-driven Ebola virus minigenome system as an advanced tool for antiviral drug screening. Antiviral Res. 2017;146:21-27.

6. Glanzer JG, Byrne BM, McCoy AM, James BJ, Frank JD, Oakley GG. In silico and in vitro methods to identify ebola virus VP35-dsRNA inhibitors. Bioorganic Med Chem. 2016;24(21):5388-5392.

7. Ilkhani H, Farhad S. A novel electrochemical DNA biosensor for Ebola virus detection. Anal Biochem. 2018;557(May):151-155.

8. Kadanali A. An overview of Ebola virus disease. North Clin Istanbul. 2016;2(1):81-86. doi:10.14744/nci.2015.97269

9. Tchesnokov EP, Raeisimakiani P, Ngure M, Marchant D, Götte M. Recombinant RNADependent RNA Polymerase Complex of Ebola Virus. Sci Rep. 2018;8(1):1-9.

10. Sievers F, Wilm A, Dineen D, et al. Fast, scalable generation of high-quality protein multiple sequence alignments using Clustal Omega. Mol Syst Biol. 2011;7(539).
11. Higgins DG, Sharp PM. CL U S T AL : a package for performing multiple sequence alignment on a microcomputer. 1988;73:237-244.

12. Larkin MA, Blackshields G, Brown NP, et al. Clustal W and Clustal X version 2.0. Bioinformatics. 2007;23(21):2947-2948.

13. Gouet P, Courcelle E, Stuart DI, Métoz F. ESPript: analysis of multiple sequence alignments in PostScript. Bioinformatics. 1999;15(4):305-308.

14. Gouet P, Robert X, Courcelle E. ESPript/ ENDscript: Extracting and rendering sequence and 3D information from atomic structures of proteins. Nucleic Acids Res. 2003;31(13):3320-3323.

15. Kabsch W, Sander C. Protein Secondary Structure: Pattern Recognition of Hydrogen-Bonded and Geometrical Features. 1983;22:2577-2637.

16. Frishman D, Argos P. Knowledge based protein secondary structure assignment. Proteins Struct Funct Bioinforma. 1995;23(4):566-579.

17. Elfiky AA, Elshemey WM, Gawad WA, Desoky OS. Molecular modeling comparison of the performance of NS5b polymerase inhibitor (PSI7977) on prevalent HCV genotypes. Protein J. 2013;32(1):75-80.

18. Kumar S, Nei M, Dudley J, Tamura K. MEGA: A biologist-centric software for evolutionary analysis of DNA and protein sequences. Brief Bioinform. 2008;9(4):299-306.

(Recieved 28/9/2019; accepted 13/10/2019) 


\section{لدراسة التحليل التطورى والتسلسلى لأنزيم البلمره الرنا المقتدة على الرنا لفيرس الإيبولا

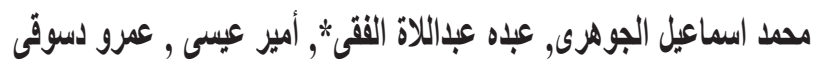

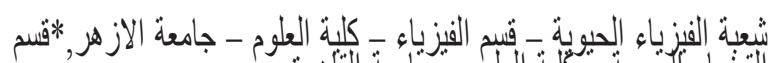

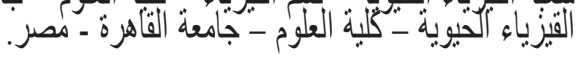

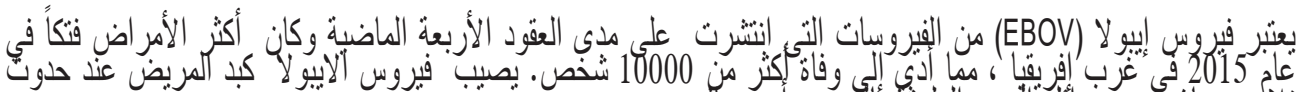

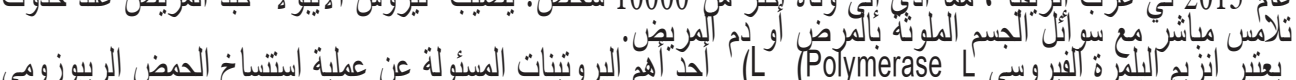

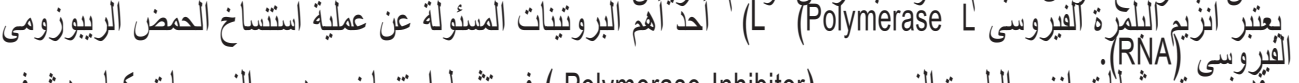

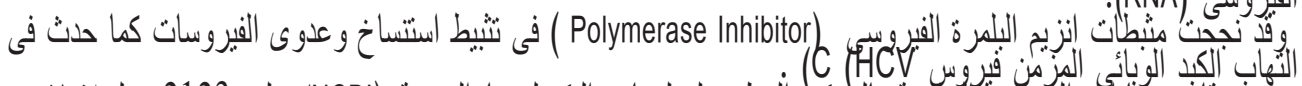

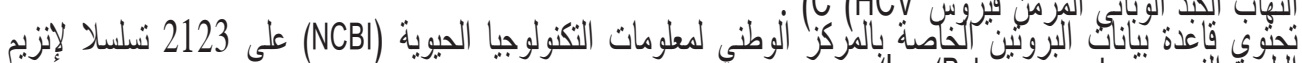

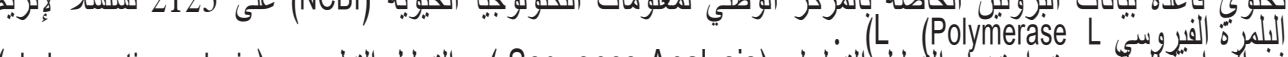

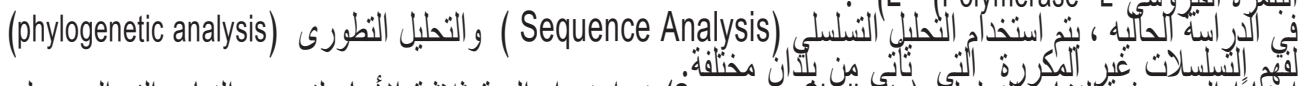

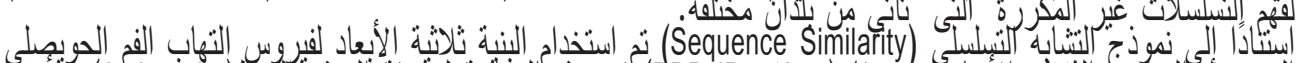

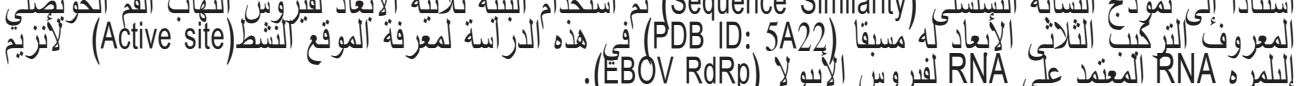

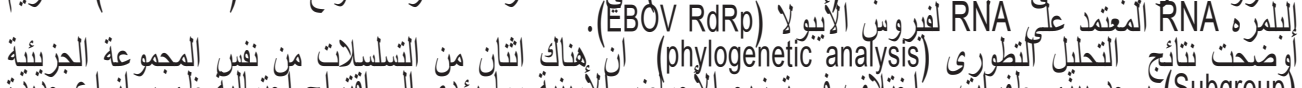
(Subgroup)

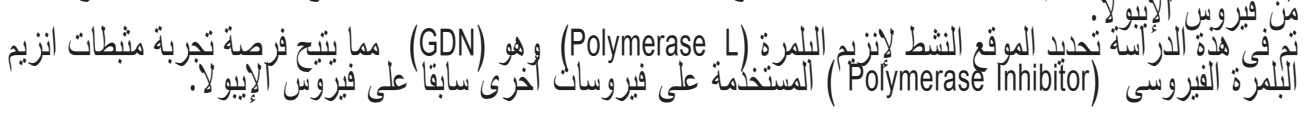

